characteristic X-ray emission energies.

I placed one of the remaining small crystals of aPP on a sample stub to air dry and to my surprise it did not disintegrate. After coating it with carbon, I examined the sample under vacuum in the microscope and it remained crystalline in appearance. I recorded EDAX spectra over a 400-s time period and observed a peak at $8.63-\mathrm{KeV}$ characteristic of the $K_{a}$ line for zinc. The addition of zinc acetate to the crystallization experiments resulted in much larger crystals, indicating that the original scavenged zinc must have been limiting ${ }^{2}$. The resulting high-resolution $\mathrm{X}$-ray analysis indicated the key role this divalent ion played in forming

\section{Expanding family}

SIR-We have recently described' a family of six fungal genes involved in mitosis and the regulation of RNA synthesis whose protein products have in common a repetitive sequence motif of 34 amino acids (tetratricopeptide repeat or TPR). Zhang et al. (manuscript in preparation) subsequently found 16 tandem TPR sequences in the crooked neck ( $\mathrm{crn}$ ) gene involved in neurogenesis in the fruitfly Drosophila. We have now identified two additional family members in the yeast Saccharomyces cerevisiae.

The product of one gene, first cloned in 1983 (refs 2, 3), is a protein of relative molecular mass $\left(M_{\mathrm{r}}\right) 70,000$ and was used for studying the targeting of mitochondrial proteins encoded in the nucleus. The 41 amino acids at the amino terminus

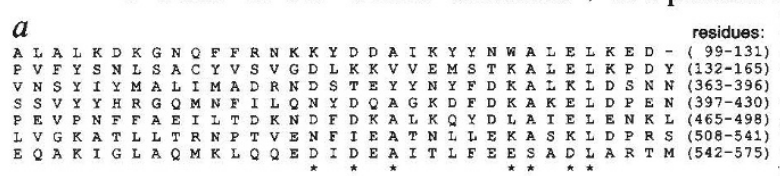
$b$

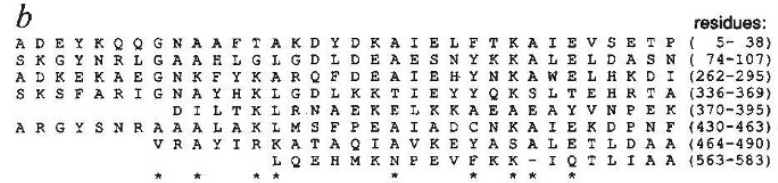

$c$ $B$

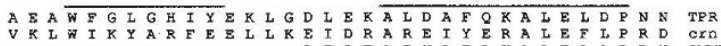

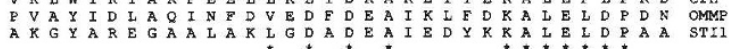

Internally repetitive sequences and their statistical significance were identified and characterized by standard methods as described in ref. 1. The $M_{r} 70,000$ protein and STI1 have intra-sequence comparison scores of 7.09 and $12.37 \mathrm{~s}$.d. units, respectively; intersequence comparisons with other members of the TPR family yielded scores ranging from 3.44 to 12.43 s.d. units. Only the repeats of highest significance are shown. Starred columns, the most highlyconserved positions in the aligned sequences. The original TPR motif was based on 47 repeats from five different proteins and showed two more highly conserved subdomains labelled $A$ and $B$ and represented by the sequences W...LG..Y and A...(Y/F)..A...P, respectively (ref. 1) The CDC subset of TPR proteins includes $C D C 23, C D C 16$ (S. cerevisiae), nuc2 ${ }^{+}$(S. pombe) and bimA (A. nidulans); the RNA regulation subset includes SKI3 and SSN6 (S. cerevisiae). To facilitate comparisons with new candidate TPR genes, a file containing all known TPR protein sequences, as well as suggestions for quantitative comparisons, is available from M.S.B. motif is maintained but there are different patterns of sequence conservation in $\mathrm{A}$. We believe that TPR genes have evolved different functions based both on divergence of TPR sequences and also on the acquisition of separate, unrelated functional domains such as the targeting/ anchoring sequence in the $M_{\mathrm{r}} 70,000$ outer mitochondrial membrane protein and the zeste-like domain in SSN6 (ref. 1). Consistent with this idea, we have now found that single amino-acid substitutions in $C D C 23$, which cause a temperature-sensitive phenotype, cluster into two separate domains: the carboxy-terminal TPR sequence block and an amino-terminal domain of unique, unrelated sequence.

\section{National Center for Biotechnology} MARK S. BOGUSKI

Information.

National Library of Medicine,

National Institutes of Health,

Bethesda, Maryland 20894, USA

ROBERT S. SIKORSKI

PHILIP HIETER

Department of Molecular Biology and Genetics,

Johns Hopkins University School of Medicine,

Baltimore, Maryland 21205, USA

Department of Biochemistry,

MARK GOEBL

Indiana University School of Medicine,

Indianapolis,

Indiana 46202, USA

1. Sikorksi, R. S., Boguski, M. S., Goebl, M. \& Hieter, P. Cell 60, 307-317 (1990)

2. Hase, T., Riezman, H., Suda, K. \& Schatz, G. EMBO J. 2169-2172 (1983)

3. Riezman, H. et al. EMBO J. 2, 2161-2168 (1983).

4. Nicolet, C. M. \& Craig, E. Molec. cell. Biol. 9, 3638-3646 (1989).

5. Hirano, T., Kinoshita, N., Morikawa, K. \& Yanagida, M. Cell 60, 319-328 (1990). Again there are internal TPR sequences, but of variable length, in an arrangement resembling that of the product of SKI3 gene involved in RNA regulation.

The inferred amphipathic helical structure of the TPR unit ${ }^{1,4}$ suggests several possible functions, including proteinlipid interaction and the mediation of protein dimerization. Our recent (unpublished) finding of intragenic complementation between temperature-sensitive alleles of $C D C 23$ by mutations in different TPRs suggests that protein-protein interactions are necessary for $C D C 23$ function.

In our original TPR dataset $^{1}$, we identified two more highly conserved subdomains, $\mathrm{A}$ and $\mathrm{B}$, corresponding to the 'hole' and 'knob' in the repeat-repeat interaction model of $n u c 2^{+}$function ${ }^{4}$. In the new examples, the B sub-
SIR-If there is unease with the idea of a defined moment for the Big Bang, with no temporal predecessor, why not adopt E. A. Milne's logarithmic proposal, where $\tau=t \log \left(t / t_{0}\right)+t_{\mathrm{o}}$, with $\tau$ as dynamical time and $t$ as conventional time (Proc. $R$. Soc. A158, 324-348; 1937)? As Milne remarks, the epoch of creation $t=0$ on the kinematic scale is measured by $\tau=-\infty$ on the dynamical scale.

Indeed, more generally there is a case for thinking of time in proportionate rather than in arithmatically progressive terms. In sensory physiology, strength of sensation is usually proportional to $\log$ (stimulus); in pharmacology, drug effect is usually proportioned to $\log ($ dose $)$; and on a wider canvas, wage negotiations, inflation, growth and what-not, are almost always expressed in proportionate terms. Why should the philosophy of time restrict itself to an unnatural arithmetical scale?

13 Staverton Road,

W. D. M. PATON

Oxford OX2 6XH, UK

\section{Proportional time}

